\title{
ZUR VERTEILUNG DER REIMSTÄBE IN DER ALLITERIERENDEN LANGZEILE.
}

Der alliterierende vers ist bisher, soweit ich sehe, eigentlich immer nur als form fur grammatisch verbundene worte verschiedener tonstärke betrachtet worden, nicht als klinstlerisches gewand von sätzen. Wäre der stabvers nur ein mechanisch gefulltes - sei es rhythmisches oder metrisches - schema, in der uberlieferung erstarrt wie etwa der mittelalterliche hexameter, dann wäre sein rascher untergang leicht begreiflich. Aber es fragt sich doch, ob die klinstlerische wirkung, die der eigentumliche bau der germanischen langzeile von anfang an hatte, in den erhaltenen literaturversen ganz verloren ist; ob der grundsätzliche unterschied der ersten und zweiten halbzeilen wirklich dem texte nicht mehr zu gute kam oder vom texte nicht mehr bedingt wurde. Die älteren verse umfassten wol - wie die des goldenen hornes - ein syntaktisctes ganzes, entstanden sie doch wol meist einzeln fir sich und erst eine fortgeschrittenere kunstubung verband sie in grösserer zahl. Nunmehr war aber durch deu inhalt wie durch den geschmack durchbrechung der alten regel geboten. Je umfangreicher eine dichtung wurde, desto häufiger stellte sich durchkreuzung der sinnabschnitte und der rhythmischen fugen ein. Vielleicht durfen wir schon aus dem umstand, dass die deutschen verse weniger satzschltusse in die cäsur fallen lassen, schliessen, dass hier lange epen nicht vorhanden waren. Ebenso kennen die ältesten nordischen dichtungen, die sämmtlich kurzen umfanges sind, syntaktische pausen nur am versschluss. In England durfen wir frcilich nicht die dichtungen, in denen sinnespausen nur (oder ganz uberwiegend) an den versschluss fallen, fur absolut älter erklären. Hier geht ältere und neuere art neben 
einander her. Alt ist nun sicher die stellung des letzten stabes am anfang der zweiten halbzeile. Wir mllssen uns vorstellen, dass der den vers fullende satz nach einer letzten (böchsten) erhebung sich bis zum ende hin senkte ${ }^{1}$ ), dass seine erste bälfte - die erste balbzeile - dagegen eine oder zwei erhebungen enthielt und sich nicht bis zu der tiefe des versschlusses herab bewegte. Das auf- und abwogen in der ersten halbzeile gibt ihr einen besonderen reiz. Dass auch in ihr einem gipfel zugestrebt wurde ist wol unursprunglich, obwol sich denken liesse, dass von anfang an die stärkegrade der vier hebungen sich annähernd verhielten wie $1: 2 \mid: 3: 1$. War einmal die scharfe hervorhebung eines stabes der 1 . hz. grundsätzlich als kunstmittel zugestanden, so wird der regel nach der bau des satzes, der sprachliche inhalt, den ausschlag gegeben haben, ob der stab auf die erste oder zweite hebung verlegt wurde. Sievers scheint (Altg. metr. $\S 9,5$ ) den stab an erster stelle für die (altererbte?) legel zu halten. Nachdem sinnabschnitte in der cäsur zugelassen waren, näherte sich die erste halbzeile im tonfall der zweiten, wie umgekehrt die fortdauernde erhebung am versschluss die zweite hz. der ersten ähnlich gemacht habeu wird. Aebnlich, nicht gleich, solange das ideale schema neben der augenblicklichen ausfullung im bewusstsein blieb. Sagen uns nun unsere denkmäler etwas hierliber? Ich glaube ja. Insofern ist das schema - und bis zu einem gewissen grado der vortrag - der alten überlieferung treu geblieben, als regelmässig die zweite balbzeile nur einen stab und regelmässig am anfang erhielt. Aber die rucksicht auf den satzbau und tonfall dringt doch kräftiger durch als man bisher anzunehmen scheint.

Ist es richtig, dass der versschluss annähernd die stärkeabstufung 3:1 erhielt, weil der satzschluss sie hatte, so ist zu erwarten, dass auch in der ersten balbzeile, wenn sie miteiner sinnespause schloss, diese ordnung sich geltend machte, und dass umgekehrt, wenn der versschluss in die mitte eines satzes fiel, hier die absteigende linie sich in eine aufsteigende oder

1) Mit einer kleinen unterbrechung durch die letzte hebung, s. R. M. Meyer, Grundlagen des mhd. strophenbanes s. $20 \mathrm{ff}$. uber den rhythmus deutscher sätze. 
auf- und abwogende wandelte. Das erstere lässt sich am Béowulf beweisen, das letztere vielleicht wahrscheinlich machen.

Als sinnespausen die mit senkung der stimme (oder wenn man will abnahme der sprechenergie) verbunden sein durften, betrachte ich diejenigen, bei welchen unsere herausgeber punkte setzen; vor semikolon nehme ich solche pausen nur an, wenn sie einschnitte zwischen zwei grösseren ganzen bilden, vor allem wenn die satzform hinter dem kolon wechselt, neue subjecte eintreten. Ich habe dabei eher fälle, die $f \mathfrak{r}$ meine regel sprächen, ausgeschlossen als eingerechnet. $\mathrm{Da}$ finden sich nun in den ersten 1000 versen etwa 123 halbverse mit doppelalliteration, etwa 48 mit alliteration auf der ersten hebung; vielleicht ein vers (301) mit all. auf der zeiten. Dieser heisst

Gewiton him pá féran; flota stille bád.

Wulker hat kolon statt semikolon, Heyne parenthese, Holder kolon.

In den folgenden 2100 versen steht vor sinneseinschnitten, von doppelalliteration abgesehen, etwa $90 \mathrm{mal}$ alliteration auf der ersten hebung und nur drei mal auf der zweiten, nämlich

1232 Éode pá tó setle. bǽr wæs symbla cyst

1573 hwearf pá be wealle; wǽpen hafenade

2298 on pǽm wéstenne. Hwæðre hilde zefeb.

Hier könnte im ersten fall pór relativum sein; im zweiten $h w$ - mit $n$ alliterieren (wie im folgenden und vielleicht auch 1601), im dritten ist der text von verschiedenen herausgebern schon lange beanstandet worden: es fehlt wol wees am anfang oder ende. $\mathrm{Zu}$ den drei versen käme etwa noch

1600 Đá cóm nón dæzes; næs ofzéafón;

so Wyatt, audere baben als interpunction doppelpunkt; der vers wird ubrigens von Sievers zum typus $C$ gerechnet werden; bei 3074

Næs hē goldhwæt; gearwor hæfde

ist stärkere interpunction an und für sich $\mathrm{zweifelhaft}$ und der text unsicher; obige lesung steht bei Wyatt gegen die hs. (die hwoete hat; so oder hwoetes die tubrigen herausgeber). Die ausnahmen von der regel: 'vor sinnespause nie stab auf der zweiten hebung allein' schrumpfen so auf eine nichtssagende zahl zusammen. Bedenken wir nun noch, dass sonst in ersten halbzeilen alliteration auf zweiter hebung nicht selten vorkommt: 
in den ersten 200 versen des Beowulf etwa 14 sichere fälle, so düren wir zufall für ausgeschlossen ansehen.

Es ist eine anziehende aufgabe, dem nachwirken des alten kunstprincipes weiter zu folgen. Ich muss es anderen tiberlassen, bemerke aber, dass z. b. der Andreas zum Beowulf stimmt; ich habe bei einer eiligen musterung nur drei ausnahmen gefunden:

275 on pǽre mǽzðe: bið ðē meorð wið zod. .

1116 hréow rícsode. pá wæs rinc maniz... hier hat schon Grein réon eingesetzt; endlich

1432 nis pe e tó frécne: ic pe friðe healde ...

der vers ist seit langem ein stein des anstosses.

Schwieriger liegt die beurteilung des zweiten halbverses. Ein teil meines materials ist nämlich nicht ohne weiteres verwendbar. Zwar die doppelstäbe des Beowulf an stellen wo der versschluss mitten in den satz fällt, wie 884. 1151. 2920 sind nicht wegzuleugnen, aber sie beweisen nicht viel wegen ihrer geringen zabl. Wichtiger scheint mir schon die zweifache (oft auch gekreuzte) alliteration in versen ohne sinnabschnitt; sie ist verhältnismässig häutig: so 32 pér aet hýठ̀e stód hrinzedstefna, ähnlich 88. 209. 237. 374. 901. 907. 1131. 1203. 1262. 1611. 1652. 1705. 1727. 1732. 1849. 1910. 1937. 1939. 1968. 2020. 30. 67. 91. 21598, 70. 2223. 35. 61 (?).66. 72 (?). 2337. 97. 2479 etc., denen nur ganz verschwindende beispiele von zwiefacher alliteration vor grösserer interpunction gegenlaber stehen, so in den ersten 2500 versen 2162. 2186. 2311, von denen der letztere nach Sievers' auffassung gar nicht hierher gehören w (urde. $\left.{ }^{1}\right)$ Wirklich alliteration an zweiter stelle allein zeigen sicher die verse $\left.{ }^{2}\right) 682$ peah $\partial e$ he róf sie, 930 á moz zod wyr-

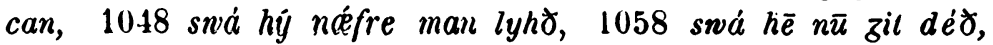
1534 Swá sceal man dón und vielleicht noch ein oder zwei verse. Viel zu wenig, um irgend etwas zu beweisen, zumal noch einige verse mit entschiedenem sinnabschluss wie 2801 ne maz ic hér lenz wesan zur not ebenso, d. b. mit dem stab auf der zweiten hebung gelesen werden können. Nun gibt es

1) Eine andere eigenheit zweiter halbzeilen ohne sinnessbschluss hat Kaluza, Altengl. versbau 1,93 behandelt, das hintibergreifen der alliteration von einem vers in den andern.

2) So wie sie sind! 
allerdings noch dutzende von versen, die nach meinem gefühl hieher gehören, nämlich die von der form

und vor allem

Ic eom Hrózzáres (335)

pæt hie sint wilcuman (388).

Ich kann die zerschneidung der composita als rhythmisches princip am versschluss so wenig naturlich finden als am anfang (etwa 2282 frioðowcére bced) und sehe keinen zwingenden grund, paet hie in béorsele (482) anders zu lesen als foet hine on ylde (22, s. Beitr. 10, 284). Ich kann den gegensatz, in welchen bei Sievers' betonungsweise die zwei compositionsglieder treten, nur verstehen, wenn sie die zwei wesentlichen bestandteile des verses siud, wie etwa in $\mathbf{\nabla} .771$ pot se winsele, 801 pone synscaðan. Sonst halte ich die betonung wie sie z. b. im Nibelungenlied an der Bartsch'schen stelle sich so oft findet (hêrlicher), d. b. unnittelbare unterordnung des zweiten gliedes unter das erste, für geboten. In versen wie wisle pám áhlácan (646) kann wiste schwächer betont sein als láe und doch hebung, sobald es nur in seiner region vorherscht und lác in seiner - der der höchsten erhebung des verses - untergeordnet ist. Doch ich kann nicht hoffen ohne sehr weit auszuholen meine auffassung der von Sievers gegentiber zu rechtfertigen und muss deshalb fir diesmal darauf verzichten, dem zweiten halbvers eine ähnliche stilistische heweglichkeit des hauptstabes nachzuweisen wie dem ersten.

WÜRZBURG, september 1894 . $\quad$ O. BRENNER.

\section{ZUM RHYTHMUS DER NIBELUNGEN- UND GUDRUNSTROPHEN.}

Dass eine rhythmische reibe von verschiedenen verschieden aufgefasst, infolgedessen verschieden vorgetragen und nachgebildet werden kann ist einleuchtend und jungst von Sievers an der ïberlieferung der Marienlieder schön nachgewiesen. Auch an den Nibelungen zeigt sich ein wandel der rhythmischen 